\title{
Presence of Heat on an Infinite Plate with a Curvilinear Hole Having Two Poles
}

\author{
F. S. Bayones \\ Math. Dept., Faculty of Science, Taif University, Taif, KSA \\ Email: f.s.bayones@hotmail.com
}

Received 6 March 2015; accepted 25 May 2015; published 28 May 2015

Copyright (C) 2015 by author and Scientific Research Publishing Inc.

This work is licensed under the Creative Commons Attribution International License (CC BY).

http://creativecommons.org/licenses/by/4.0/

(c) (i) Open Access

\begin{abstract}
In the present paper Cauchy integral methods have been applied to derive exact and expressions for Goursat's function for the first and second fundamental problems of isotropic homogeneous perforated infinite elastic media in the presence of uniform flow of heat. For this, we considered the problem of a thin infinite plate of specific thickness with a curvilinear hole where the origins lie in the hole is conformally mapped outside a unit circle by means of a specific rational mapping. Moreover, the three stress components $\sigma_{x x}, \sigma_{y y}$ and $\sigma_{x y}$ of the boundary value problem in the thermoelasticity plane are obtained. Many special cases of the conformal mapping and four applications for different cases are discussed and many main results are derived from the work.
\end{abstract}

\section{Keywords}

An Elastic Plate, Presence of Heat, Curvilinear Hole, Cauchey Method, Conformal Mapping

\section{Introduction}

The boundary value problems for isotropic performed plates have been discussed by several authors. Some authors used Laurant's theorem [1]-[8] to express each complex potential as a power series, and others [9]-[16] used complex variable method of Cauchy integral. The extensive literature on the isotropic is now available and we can only mention a few recent interesting investigations in refs [17]-[26].

In thermoelastic problems for elastic media, the first and second boundary value problems are equivalent to two finite analytic functions $\phi(z)$ and $\psi(z)$ of one complex argument $z=x+i y$. These functions must satisfy the boundary condition,

$$
K \phi_{1}(t)-t \overline{\phi_{1}^{\prime}(t)}-\overline{\psi_{1}(t)}=f(t)
$$


where $t$ denotes the affix of a point on the boundary. In terms of $z=c \omega(\zeta), c>0, \omega^{\prime}(\zeta)$ does not vanish or become infinite for $|\zeta|>1$, where the infinite region is outside a unit circle $\gamma$. For the first fundamental boundary value problems or the called stress boundary value problems, $K=-1, f(t)$ is a given function of stress. While for $K=k>1, f(t)$ is a given function of displacement called the thermal conductivity; we have the second fundamental boundary value problems called the displacement boundary value problems.

The complex potential functions $\phi_{1}(t)$ and $\psi_{1}(t)$ take the following forms, see [23]

$$
\phi_{1}(\zeta)=-\frac{X+i Y}{2 \pi(1+k)} \ln \zeta+c \Gamma \zeta+\phi(\zeta)
$$

and,

$$
\psi_{1}(\zeta)=k \frac{(X-i Y)}{2 \pi(1+k)} \ln \zeta+c \Gamma^{*} \zeta+\psi(\zeta),
$$

where, $X, Y$ are the components of the resultant vector of all external forces acting on the boundary and $\Gamma, \Gamma^{*}$ are complex constants. Generally the two complex functions $\phi(\zeta)$ and $\psi(\zeta)$ are single value analytic functions within the region outside the unit circle $\gamma$ and $\phi(\infty)=0, \psi(\infty)=0$. In [27], Muskhelishvili used the rational mapping,

$$
z=c\left(\zeta+m \zeta^{-1}\right), c>0, m \text { is real number, }
$$

for solving the problem of stretching of an infinite plate weakened by an elliptic hole. Sokolonikoff [3] used the same rational mapping of Equation (4) to solve the problem of elliptical ring, where the Laurant's theorem is used. This transformation of Equation (4) conformally maps the infinite domain bounded internally by an ellipse onto the domain outside the unit circle $|\zeta|=1$ in the $\zeta$-plane. The application of the Hilbert problem is used by Muskhelishvili [27] to discuss the case of a stretched infinite plate weakened by a circular cut. England [1] considered an infinite plate which is weakened by a hypotrochoid hole, conformally mapped onto a unit circle $|\zeta|=1$ by the transformation mapping

$$
z=c\left(\zeta+m \zeta^{-n}\right), \quad c>0,0 \leq m<\frac{1}{n}
$$

where $z^{\prime}(\zeta)$ does not vanish or become infinite outside the unit circle $\gamma$, and solve the boundary value problems.

The main reason for interest in this mapping is that the general shape of the hypotrochoids is curvilinear polygons, for $n=1$ ellipse, for $n=2$ a curvilinear triangle, for $n=3$ a curvilinear square... etc. In previous papers (Abdou et al. [9]-[15] [28] [29]), the complex variable method has been applied to solve the first and second fundamental problems for the same domain of the infinite plate with general curvilinear hole $c$ conformally mapped on the domain outside a unit circle by using respectively, the rational mapping functions

$$
\begin{aligned}
& z=c \frac{\zeta+m \zeta^{-1}}{1-n \zeta^{-1}}, \quad c>0,|n|<1 \\
& z=c \frac{\zeta+m_{1} \zeta^{-1}+\ell n_{2} \zeta^{-2}}{1-n \zeta^{-1}}, \quad c>0,|n|<1 \\
& z=c \frac{\zeta+m \zeta^{-\ell}}{1-n \zeta^{-\ell}}, \quad c>0,|n|<1, \ell=1,2, \cdots, p
\end{aligned}
$$

and

$$
z=c \frac{\zeta+m_{1} \zeta^{-1}+m_{2} \zeta^{-2}+m_{3} \zeta^{-3}}{1-n \zeta^{-1}}, \quad c>0,|n|<1 .
$$

In this paper, the complex variable method has been applied to solve the first and second fundamental prob- 
lems for the same previous domain of the infinite plate with a general curvilinear hole $C$, with three poles and presence of heat, conformally mapped on the domain outside a unit circle $\gamma$ by the rational mapping functions

$$
z=c \omega(\zeta)=c \frac{\zeta+m \zeta^{-\ell}}{\left(1-n_{1} \zeta^{-\ell}\right)\left(1-n_{2} \zeta^{-\ell}\right)}, \quad c>0, n_{1} \neq n_{2}
$$

where $c>0, \ell=1,2, \cdots, p, m$ and $n$ are real parameters restricted such that $z^{\prime}(\zeta)$ does not vanish or become infinite outside the unit circle $\gamma$. Consider a heat $\Theta=q y$ flowing uniformly in the direction of the negative $y$-axis, where the increasing temperature $\Theta$ is assumed to be constant across the thickness of the layer, i.e. $\Theta=\Theta(x, y)$, and $q$ is the constant temperature gradient. The uniform flow of heat is distributed by the presence of an insulated curvilinear hole $C$. The heat equation satisfies the relations,

$$
\begin{gathered}
\nabla^{2} \Theta=0, \quad \nabla^{2}=\frac{\partial^{2}}{\partial x^{2}}+\frac{\partial^{2}}{\partial y^{2}} \\
\frac{\partial \Theta}{\partial n}=0, \quad r=r_{0}
\end{gathered}
$$

where $n$ is the unit vector perpendicular to the surface.

Neglecting the variation of the strain and the stress with respect to the thickness of the layer, the thermoelastic potential function $\Phi$ satisfies the formula (see [14]),

$$
\nabla^{2} \Phi=(1+v) \alpha \Theta
$$

where $\alpha$ is a scalar which presents the coefficient of the thermal expansion and $v$ is Poisson's ratio. Assume that the force of the layer is free of applied loads.

In this case, formula (1) for the first and second boundary value problems respectively takes the following forms,

$$
\begin{gathered}
\phi_{1}(t)+t \overline{\phi_{1}^{\prime}(t)}+\overline{\psi_{1}(t)}=\frac{\partial \Phi}{\partial x}+i \frac{\partial \Phi}{\partial y}+\frac{1}{2 G} \int_{0}^{s}[i X(s)-Y(s)] \mathrm{d} s+c \\
k \phi_{1}(t)-t \overline{\phi_{1}^{\prime}(t)}-\overline{\psi_{1}(t)}=u+i v-\frac{\partial \Phi}{\partial x}-i \frac{\partial \Phi}{\partial y}
\end{gathered}
$$

where the applied stresses $X(s)$ and $Y(s)$ are prescribed on the boundary of the plane; $s$ is the length measured from an arbitrary point; $u$ and $v$ are the displacement components; $G$ is the shear modulus and $\Phi$ represents the thermoelastic potential function. Also, here the applied stresses $X(s)$ and $Y(s)$ must satisfy the following (see [14]),

$$
\begin{aligned}
& X(s)=\sigma_{x x} \frac{\mathrm{d} y}{\mathrm{~d} s}-\sigma_{x y} \frac{\mathrm{d} x}{\mathrm{~d} s} \\
& Y(s)=\sigma_{y x} \frac{\mathrm{d} y}{\mathrm{~d} s}-\sigma_{y y} \frac{\mathrm{d} x}{\mathrm{~d} s}
\end{aligned}
$$

where $\sigma_{x x}, \sigma_{x y}$ and $\sigma_{y y}$ are the components of stresses which are given by the following relations,

$$
\begin{gathered}
\sigma_{x x}-\sigma_{y y}+2 i \sigma_{x y}=2 G\left[\frac{\partial^{2} \Phi}{\partial y^{2}}-\frac{\partial^{2} \Phi}{\partial x^{2}}+2 i \frac{\partial^{2} \Phi}{\partial x \partial y}\right]+4 G\left[\bar{z} \phi^{\prime \prime}(z)+\psi^{\prime \prime}(z)\right] \\
\sigma_{x x}+\sigma_{y y}=4 G\left[\operatorname{Re} \phi^{\prime}(z)-\lambda \Theta\right]
\end{gathered}
$$

where $\lambda=\frac{\alpha}{2}(1+v)$ is the coefficient of heat transfer.

The rational mapping $z=c \omega(\zeta)$ maps the boundary $C$ of the given region occupied by the middle plane of 
the layer in the z-plane onto the unit circle $\gamma$ in the $\zeta$-plane. Curvilinear coordinates $(\rho, \theta)$ are thus introduced into the $z$-plane, which are the maps of the polar conditions in the $\zeta$-plane as given by $\zeta=\rho \mathrm{e}^{\mathrm{i} \theta}$, $0 \leq \theta \leq 2 \pi$. By using the transformation $z=c \omega(\zeta)$, Equation (1) reduces to,

$$
K \phi_{1}(c \omega(\zeta))-\frac{\omega(\zeta)}{\overline{\omega^{\prime}(\zeta)}} \overline{\phi_{1}(c \omega(\zeta))}-\overline{\psi_{1}(c \omega(\zeta))}=f(c \omega(\zeta))
$$

the last formula represents the first and second boundary value problems in the $\zeta$-plane.

In this study, we use the rational mapping (10) to map the curvilinear hole $C$ of the infinite viscoelastic fluid layer outside a unit circle $\gamma$. Then, we use the properties of Cauchy integral to obtain the two complex potential functions (Goursat functions). After that, we determine the components of stresses. We establish many applications and special cases from this work.

\section{The Rational Mapping}

The mapping function (10) maps the curvilinear hole $C$ in z-plane onto the domain of the outside unit circle in $\zeta$-plane under the conditions that $\omega^{\prime}(\zeta)$ does not vanish or become infinite outside the unit circle $\gamma$. The following graphs give the different shapes of the rational mapping (10), see Figure 1.

\section{Method of Solution}

In this section, we use the complex variable method to obtain the two complex functions (Goursat functions) $\phi(\zeta)$ and $\psi(\zeta)$. Moreover, the three stress components $\sigma_{x y}, \sigma_{x x}$ and $\sigma_{y y}$ will be completely determined. The solution of Equation (9) is given by,

$$
\Theta=q\left[R+\frac{r_{0}^{2}}{R}\right], \quad R=\sqrt{x^{2}+y^{2}} .
$$

By substituting Equation (19) in Equation (11) and using the definition of $\nabla^{2} \Phi$ in polar coordinates the thermoelastic potential function takes the form,

$$
\Phi=\frac{(1+v) \alpha q r_{0}^{2}}{4} \ln z R^{2} .
$$

Also, the stresses components can be adapted in the forms,

$$
\begin{gathered}
\sigma_{x x}=2 G\left[-\frac{1}{2}\left(\frac{\partial^{2} \Phi}{\partial y^{2}}-\frac{\partial^{2} \Phi}{\partial x^{2}}+2 \lambda \Theta\right)+\operatorname{Re}\left[2 \phi^{\prime}(z)-\bar{z} \phi^{\prime \prime}(z)-\psi^{\prime \prime}(z)\right]\right] \\
\sigma_{y y}=2 G\left[\frac{1}{2}\left(\frac{\partial^{2} \Phi}{\partial y^{2}}-\frac{\partial^{2} \Phi}{\partial x^{2}}-2 \lambda \Theta\right)+\operatorname{Re}\left[2 \phi^{\prime}(z)+\bar{z} \phi^{\prime \prime}(z)+\psi^{\prime \prime}(z)\right]\right]
\end{gathered}
$$

and,

$$
\sigma_{x y}=2 G\left[\frac{\partial^{2} \Phi}{\partial x \partial y}+\operatorname{Im}\left(\bar{z} \phi^{\prime \prime}(z)+\psi^{\prime \prime}(z)\right)\right] .
$$

By using Equation (19) and Equation (20), Equations (21)-(23) becomes

$$
\begin{gathered}
\sigma_{x x}=2 G\left\{-\eta\left(z^{2}+4 z \bar{z}+\bar{z}^{2}\right) \operatorname{Im} z+\operatorname{Re}\left[2 \phi^{\prime}(z)-M(z, \bar{z})\right]\right\} \\
\sigma_{y y}=2 G\left\{\eta\left(z^{2}+4 z \bar{z}+\bar{z}^{2}\right) \operatorname{Im} z+\operatorname{Re}\left[2 \phi^{\prime}(z)+M(z, \bar{z})\right]\right\}
\end{gathered}
$$

and, 


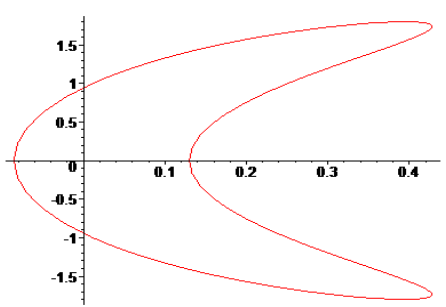

$n_{1}=-0.1, n_{2}=0.3$,

$m=-0.9, \ell=1$.

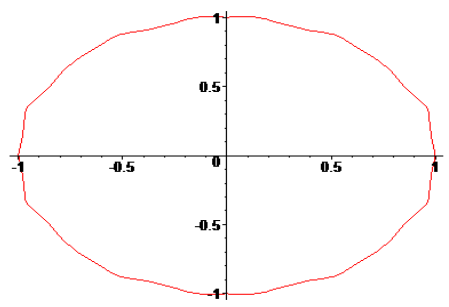

$n_{1}=-0.1, n_{2}=0.1$,

$m=-0.1, \ell=9$.

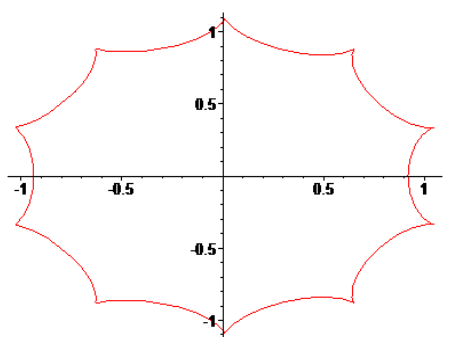

$n_{1}=-0.1, n_{2}=0.2$,

$m=-0.01, \ell=10$.

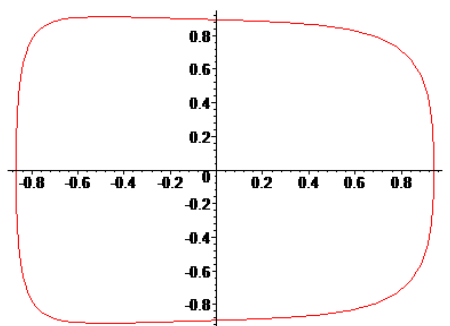

$n_{1}=0.08, n_{2}=-0.04$,

$m=-0.1, \ell=3$.

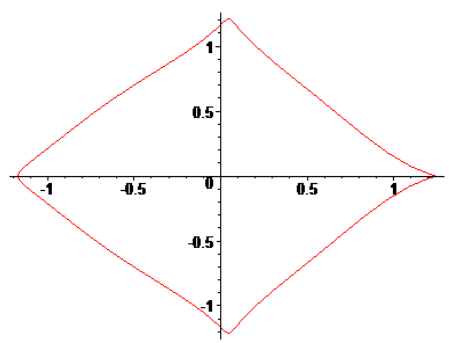

$n_{1}=0.08, n_{2}=0.1$,

$m=0.3, \ell=4$.

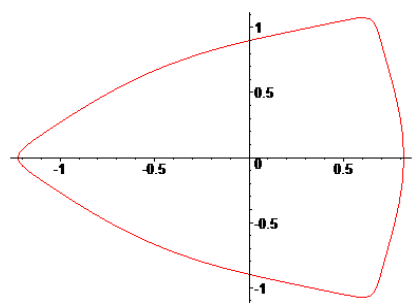

$n_{1}=-0.1, n_{2}=-0.1$,

$m=-0.01, \ell=3$.

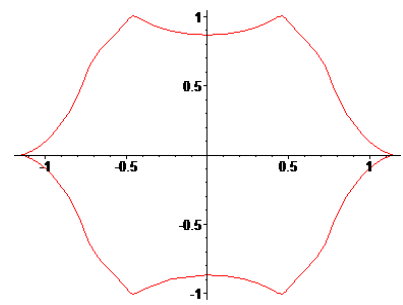

$n_{1}=-0.2, n_{2}=0.2$,

$m=0.1, \ell=5$.

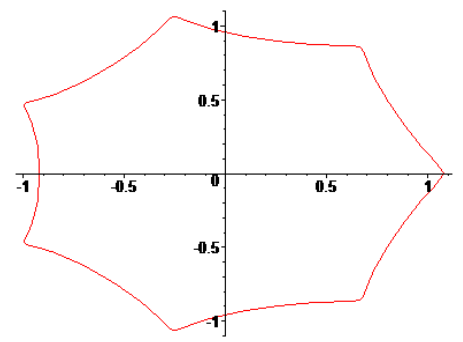

$n_{1}=0.1, n_{2}=-0.02$,

$m=-0.01, \ell=7$.

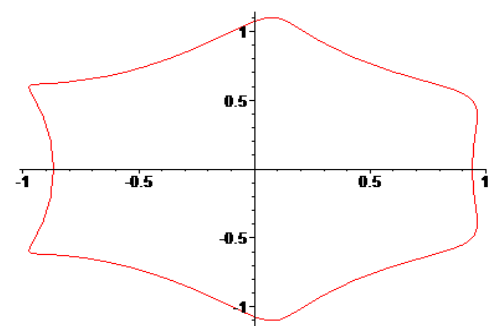

$n_{1}=0.08, n_{2}=-0.04$,

$m=-0.1, \ell=5$.

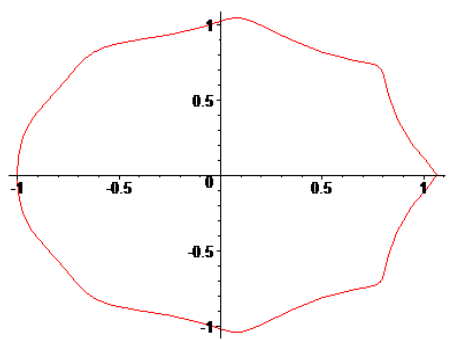

$n_{1}=0.06, n_{2}=-0.03$,

$m=0.03, \ell=8$.

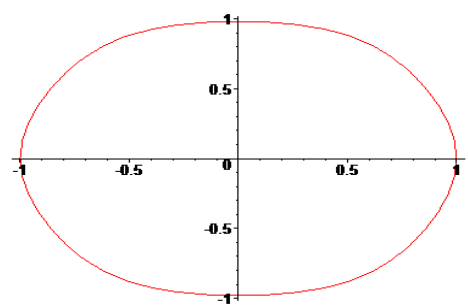

$n_{1}=-0.1, n_{2}=0.1$,

$m=-0.01, \ell=3$.

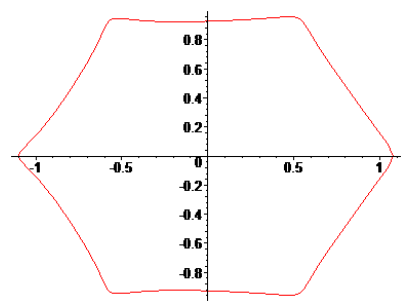

$n_{1}=0.1, n_{2}=-0.2$,

$m=-0.1, \ell=6$.

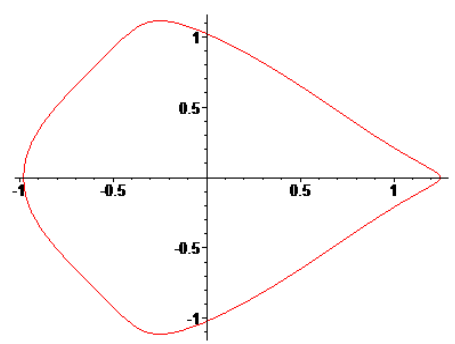

$n_{1}=0.1, n_{2}=0.02$,

$m=0.1, \ell=3$.

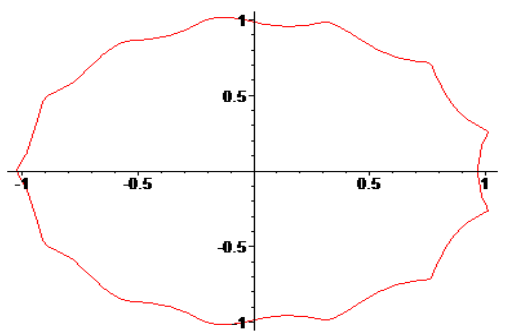

$n_{1}=-0.08, n_{2}=0.07$,

$m=-0.03, \ell=12$.

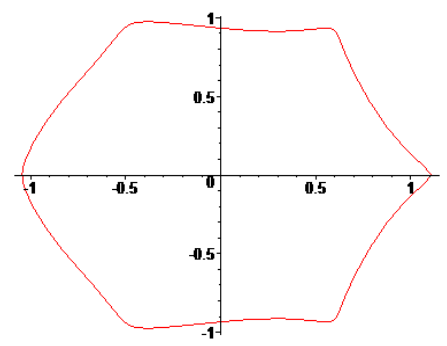

$n_{1}=-0.02, n_{2}=0.09$,

$m=0.03, \ell=6$.

Figure 1. The different shapes of the rational mapping (10). 


$$
\sigma_{x y}=2 G\left\{\eta\left(z \bar{z}-2(\operatorname{Im} z)^{2} \operatorname{Re} z+\operatorname{Im} M(z, \bar{z})\right\}\right.
$$

where,

$$
\eta=\frac{(1+v) r_{0}^{2}}{2(z \bar{z})^{2}}, \quad M(z, \bar{z})=\bar{z} \phi^{\prime \prime}(z)+\psi^{\prime}(z) .
$$

After determining the Goursat functions the components of stress are completely determined.

Using Equation (8), the expression $\frac{\omega(\zeta)}{\omega^{\prime}\left(\zeta^{-1}\right)}$ can be written in the form,

$$
\frac{\omega(\zeta)}{\omega^{\prime}\left(\zeta^{-1}\right)}=\alpha(\zeta)+\beta\left(\zeta^{-1}\right)
$$

where,

$$
\begin{gathered}
\alpha(\zeta)=\sum_{j=1}^{2} \frac{h_{j}}{\zeta^{\ell}-n_{j}} . \\
h_{j}=\frac{\left(n_{j}^{3}+m n_{j}\right)\left(1-n_{j}^{2}\right)^{2}\left(1-n_{1} n_{2}\right)^{2}}{\left(n_{j}-n_{3-j}\right)\left\{\ell\left(1-n_{1} n_{j}^{v}\right)\left(1-n_{j}^{1+v}\right)\left[3 n_{j}^{v-1}+m n_{j}^{1+v}\right]-\ell\left[2+m n_{j}^{1+v}-n_{j}^{v}\left(n_{1}+n_{2}\right)\left(1+m n_{j}^{2}\right)\right]\right\}}
\end{gathered}
$$

$v=\frac{1}{\ell}, j=1,2$ and $\beta(\zeta)$ is a regular function for $|\zeta|>1$.

Using Equation (2) and Equation (3) in Equation (18), we get

$$
K c \Gamma \zeta+K \phi(\zeta)+\frac{\omega(\zeta)}{\omega^{\prime}\left(\zeta^{-1}\right)}\left[\frac{X-i Y}{2 \pi(1+k)} \zeta-c \bar{\Gamma}-\overline{\phi^{\prime}(\zeta)}\right]+c \overline{\Gamma^{*}} \zeta^{-1}-\overline{\psi(\zeta)}=F(\zeta)
$$

using Equation (29) in Equation (31), we have

$$
K \phi(\zeta)-\alpha(\zeta) \overline{\phi^{\prime}(\zeta)}-\overline{\psi(\zeta)}-\overline{\beta(\zeta) \phi^{\prime}(\zeta)}-N(\zeta)[\alpha(\zeta)+\overline{\beta(\zeta)}]=F(\zeta)-c K \Gamma \zeta-c \bar{\Gamma}^{*} \zeta^{-1}
$$

taking $\zeta=\sigma$ for generality, we get

$$
K \phi(\sigma)-\alpha(\sigma) \overline{\phi^{\prime}(\sigma)}-\overline{\psi_{*}(\sigma)}=F_{*}(\sigma)
$$

where,

$$
\begin{gathered}
\psi_{*}(\zeta)=\psi(\zeta)+\beta(\zeta) \phi^{\prime}(\zeta) \\
F_{*}(\zeta)=F(\zeta)-c K \Gamma \zeta-c \overline{\Gamma^{*}} \zeta^{-1}+N(\zeta)[\alpha(\zeta)+\overline{\beta(\zeta)}] \\
N(\zeta)=c \bar{\Gamma}-\frac{X-i Y}{2 \pi(1+k)} \zeta
\end{gathered}
$$

and,

$$
F(\zeta)=f(c \omega(\zeta))=f(t)
$$

Assume that the function $F(\sigma)$ with its derivatives must satisfy Hölder condition. Our aim is to determine the functions $\phi(\zeta)$ and $\psi(\zeta)$ for the various boundary value problems. For this multiply both sides of Equ- 
ation (35) by $\frac{\mathrm{d} \sigma}{2 \pi i(\sigma-\zeta)}$ where $\zeta$ is any point in the interior of $\gamma$ an integral over the circle, we obtain

$$
\frac{K}{2 \pi i} \int_{\gamma} \frac{\phi(\sigma)}{(\sigma-\zeta)} \mathrm{d} \sigma-\frac{1}{2 \pi i} \int_{\gamma} \frac{\alpha(\sigma) \overline{\phi^{\prime}(\sigma)}}{(\sigma-\zeta)} \mathrm{d} \sigma-\frac{1}{2 \pi i} \int_{\gamma} \frac{\overline{\psi_{*}(\sigma)}}{(\sigma-\zeta)} \mathrm{d} \sigma=\frac{1}{2 \pi i} \int_{\gamma} \frac{F(\sigma)}{(\sigma-\zeta)} \mathrm{d} \sigma
$$

using Equations (35)-(39) in Equation (40) then applying the properties of Cauchy integral to have

$$
-K \phi(\zeta)-\frac{1}{2 \pi i} \int_{\gamma} \frac{\alpha(\sigma) \overline{\phi^{\prime}(\sigma)}}{(\sigma-\zeta)} \mathrm{d} \sigma-\frac{1}{2 \pi i} \int_{\gamma} \frac{N(\sigma) \alpha(\sigma)}{(\sigma-\zeta)} \mathrm{d} \sigma=A(\zeta)-c \overline{\Gamma^{*}} \zeta^{-1}
$$

where,

$$
A(\zeta)=\frac{1}{2 \pi i} \int \frac{F(\sigma)}{\gamma} \frac{\mathrm{d} \sigma}{(\sigma-\zeta)}
$$

The formula (41) represents the integro-differential equation of the second kind with Cauchy kernel.

To obtain the integral in terms of Equation (41) we use Equation (29) and then apply the residue theorem to have,

$$
\frac{1}{2 \pi i} \int_{\gamma} \frac{\alpha(\sigma) \overline{\phi^{\prime}(\sigma)}}{(\sigma-\zeta)} \mathrm{d} \sigma=c \sum_{j=1}^{2} \frac{h_{j} b_{j}}{n_{j}^{v}-\zeta}
$$

where $b_{j}, j=1,2$ are complex constants which can be determined.

Also,

$$
\frac{1}{2 \pi i} \int_{\gamma} \frac{N(\sigma) \alpha(\sigma)}{(\sigma-\zeta)} \mathrm{d} \sigma=\sum_{j=1}^{2} \frac{N\left(n_{j}^{v}\right) h_{j}}{n_{j}^{v}-\zeta}
$$

So, Equation (41) reduce to,

$$
-K \phi(\zeta)=A(\zeta)-c \overline{\Gamma^{*}} \zeta^{-1}+\sum_{j=1}^{2} \frac{h_{j}}{n_{j}^{v}-\zeta}\left[N\left(n_{j}^{v}\right)+c b_{j}\right]
$$

Differentiating Equation (45) with respect to $\zeta$ and using the result in Equation (44), we obtain

$$
c K b_{j}+c \Gamma^{*} n_{j}^{2 v}+h_{j} d_{j}\left(\overline{N\left(n_{j}^{v}\right)}+c \bar{b}_{j}\right)=-\overline{A\left(n_{j}^{v}\right)}
$$

where,

$$
d_{j}=n_{j}^{2 v}\left(1-n_{j}^{2 v}\right)^{-2}, j=1,2
$$

Hence, we have

$$
b_{j}=\frac{K E_{j}-h_{j} d_{j} \bar{E}_{j}}{c\left(K^{2}-h_{j}^{2} d_{j}^{2}\right)}
$$

where,

$$
E_{j}=-\overline{A^{\prime}\left(n_{j}^{-v}\right)}-c \Gamma^{*} n_{j}^{2 v}-h_{j} d_{j} \overline{N\left(n_{j}^{v}\right)}
$$

Also, from the boundary condition (35), $\psi(\zeta)$ can be determined in the form 


$$
\begin{aligned}
\psi(\zeta)= & c K \Gamma \zeta^{-1}-\frac{\omega\left(\zeta^{-1}\right)}{\omega^{\prime}(\zeta)} \phi_{*}(\zeta)+B(\zeta)-B+\sum_{j=1}^{2}\left\{\frac{h_{j} \zeta^{\ell}}{1-n_{j} \zeta^{\ell}} \phi_{*}\left(n_{j}^{-1}\right)\right. \\
& \left.+\frac{c h_{j}^{2}}{K}\left(b_{j}+N\left(n_{j}^{v}\right)\right) \delta_{j}^{1}\left(n_{j}^{v}\right)+\frac{c h_{j} \bar{\Gamma}^{*}}{K} \delta_{j}^{2}(\zeta)+\frac{h_{j}(X-i Y)}{2 \pi(1+\chi)} \delta_{3}+L_{j}\right\}
\end{aligned}
$$

where,

$$
\begin{aligned}
& B(\zeta)=\frac{1}{2 \pi i} \int_{\gamma} \frac{\overline{F(\sigma)}}{(\sigma-\zeta)} \mathrm{d} \sigma \\
& B=\frac{1}{2 \pi i} \int_{\gamma} \frac{\overline{F(\sigma)}}{\sigma} \mathrm{d} \sigma \\
& \phi_{*}(\zeta)=\phi^{\prime}(\zeta)+\overline{N(\zeta)} \\
& \overline{N(\zeta)}=c \Gamma-\frac{X+i Y}{2 \pi(1+k)} \zeta^{-1} \\
& L_{j}=\frac{h_{j}}{2 \pi i K} \int_{\gamma} \delta_{j}^{1}(\sigma) F(\sigma) \mathrm{d} \sigma
\end{aligned}
$$

and,

$$
\begin{aligned}
& \delta_{j}^{1}(\sigma)=\frac{\zeta \sigma^{\ell-2}\left[\left(1-\ell-n_{j} \sigma^{\ell}\right)(\sigma-\zeta)+\left(1-n_{j} \sigma^{\ell}\right) \sigma\right]}{\left(1-n_{j} \sigma^{\ell}\right)^{2}(\sigma-\zeta)^{2}} \\
& \delta_{j}^{2}(\zeta)= \begin{cases}n_{j}+\zeta^{-1} & \ell=1 \\
1 & \ell=2 \\
0 & \ell=3\end{cases} \\
& \delta_{3}= \begin{cases}1 & \ell=1 \\
0 & \text { otherwise }\end{cases}
\end{aligned}
$$

\section{Special Cases}

Now, we are in a position to consider several cases:

1) For $n_{1}=0$, we have the mapping $z=c\left(\zeta+m \zeta^{-\ell}\right) / 1-n \zeta^{-\ell}, \ell=1,2, \cdots, p$ discussed by Abdou and Kkhar El-din [9], see Figure 2.

2) For $n_{1}=0, \quad \ell=1$, we have the mapping $z=c\left(\zeta+m \zeta^{-1}\right) / 1-n \zeta^{1}$ discussed by El-Sirafy and Abdou [15], see Figure 3.

3) For $n_{1}=0, n_{2}=0$, we have the mapping $z=c\left(\zeta+m \zeta^{-\ell}\right), 0 \leq m \leq \frac{1}{\ell}$. The main reasons of interest in this mapping is that the general shapes of the hypotrochids are curvilinear polygons, for $\ell=1$ see Muskhelishvili's [27]. For $\ell=2$ we have a curvilinear triangle see Abdou [28]. For $\ell=3$ a curvilinear square, see Abdou and Badr [29], see Figure 4.

\section{Some Applications}

In this section, we assume different values of the given function in the first or second fundamental boundary value problems. Then, we obtain the expression of Goursat functions. After that, the components of stresses can be calculated directly. 

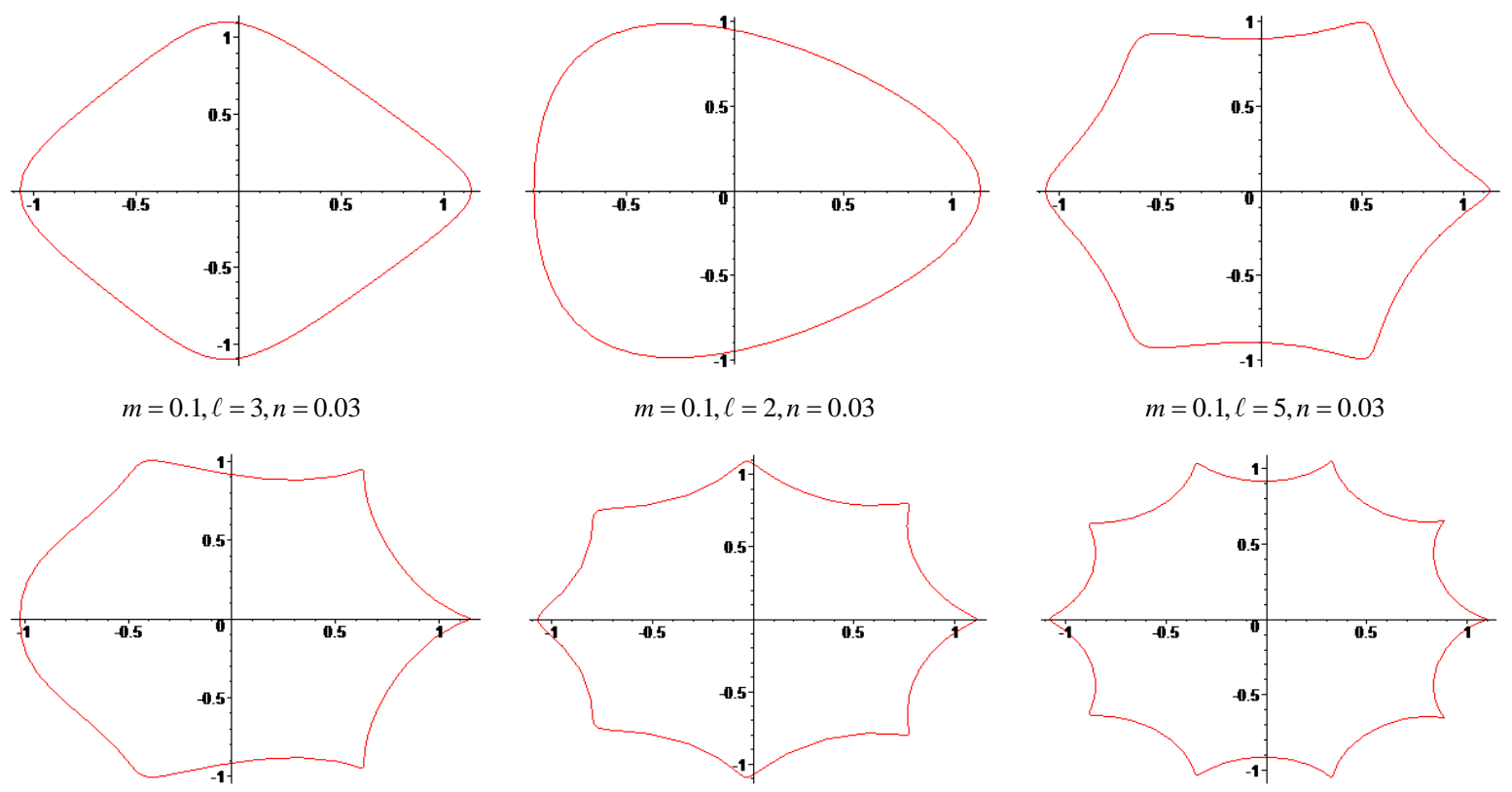

$m=0.06, \ell=6, n=0.08$

$m=0.09, \ell=7, n=0.02$

$m=0.09, \ell=9, n=0.01$

Figure 2. The different shapes of the rational mapping in (1).

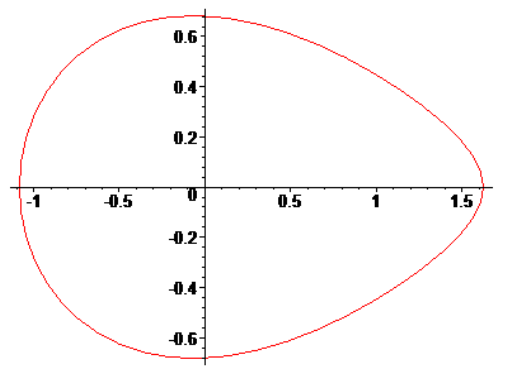

$m=0.3, \ell=1, n=0.2$

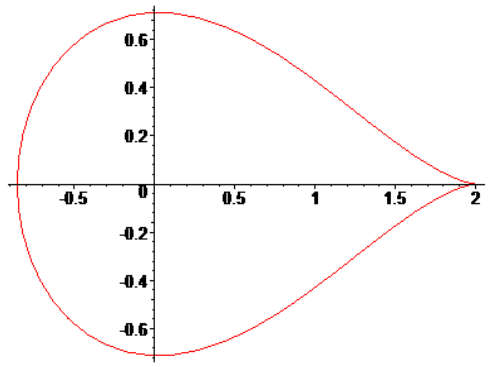

$m=0.2, \ell=1, n=0.4$

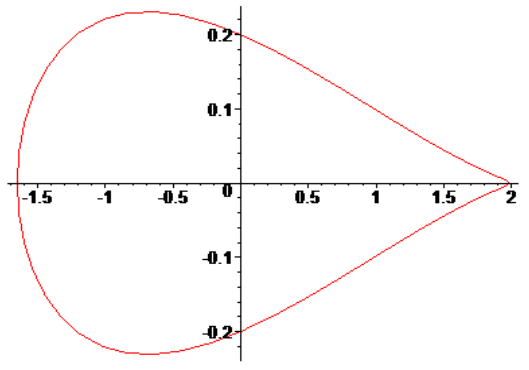

$m=0.8, \ell=1, n=0.09$

Figure 3. The different shapes of the rational mapping in (2).

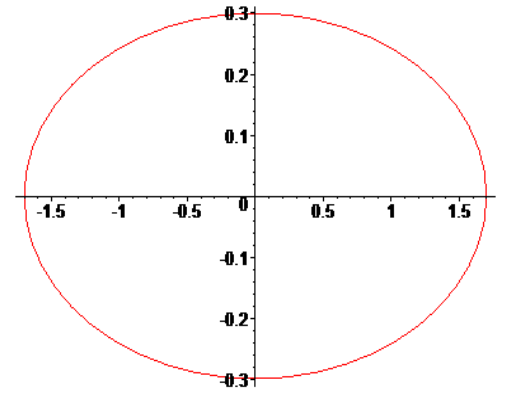

$m=0.7, \ell=1$

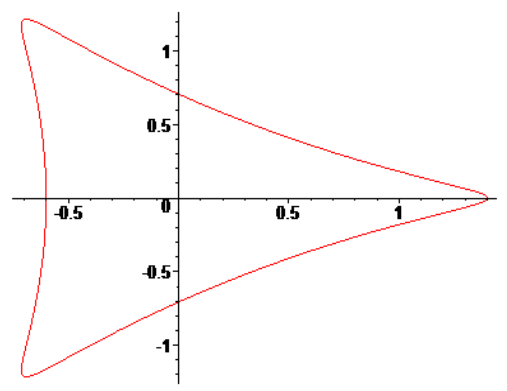

$m=0.4, \ell=2$

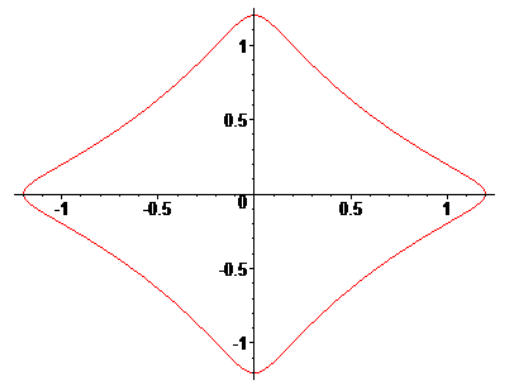

$m=0.2, \ell=3$

Figure 4. The different shapes of the rational mapping in (3).

1. Application 1: When the curvilinear centre is not allowed to rotate. At $\ell=1$, the rigid curvilinear kernel is restrained in its original position by a couple which is not sufficient to rotate the kernel.

For $K=k, \Gamma=\frac{P}{4}, \Gamma^{*}=\frac{-P}{2} \mathrm{e}^{-2 i \theta}, 0 \leq \theta \leq 2 \pi, X=Y=f=0$, 
Goursat's functions in Equations (45) and (50) take the form

$$
\begin{gathered}
-k \phi(\zeta)=\frac{c P}{2} \zeta^{-1} \mathrm{e}^{2 i \theta}+\sum_{j=1}^{2} \frac{c \tau}{n_{j}-\zeta} \\
\psi(\zeta)=\frac{c P}{4} k \zeta^{-1}-\frac{\omega\left(\zeta^{-1}\right)}{\omega^{\prime}(\zeta)} \phi_{*}(\zeta)+\sum_{j=1}^{2} \frac{h_{j} \zeta}{1-n_{j} \zeta} \phi_{*}\left(n_{j}^{-1}\right)
\end{gathered}
$$

where,

$$
\tau=\frac{p h_{j}\left(k+2 n_{j}^{2} \cos 2 \theta\right)}{4\left(k+h_{j} \alpha_{j}\right)}-\frac{i p n_{j}^{2} \sin 2 \theta}{2\left(k-h_{j} \alpha_{j}\right)}, \alpha_{j}=\frac{n_{j}^{2}}{\left(1-n_{j}^{2}\right)}, \phi_{*}(\zeta)=\phi^{\prime}(\zeta)+\frac{c P}{4} .
$$

This application discusses the first fundamental boundary value problem of an infinite layer stretched at infinity by the application of a uniform tensile stress of intensity heat in the negative direction of $y$-axis. This layer is weakened by a curvilinear hole $C$ which is free from stress.

For $n_{1}=0.09, n_{2}=0.4, m=-0.8, p=0.25$, the stress components $\sigma_{x x}, \sigma_{y y}$ and $\sigma_{x y}$ are obtained in large forms calculated by computer and illustrated in the following two cases:

1) In the thermoelasticity layer, we have the following shapes for the stress components by using the substitutions $G=0.5, q=0.1, \quad r_{0}=0.75, \alpha=0.7, v=0.7$, see Figure 5 and Figure 6 .

2) When the study is in the normal layer, we have the following shapes for the stress components, see Figure 7 and Figure 8.

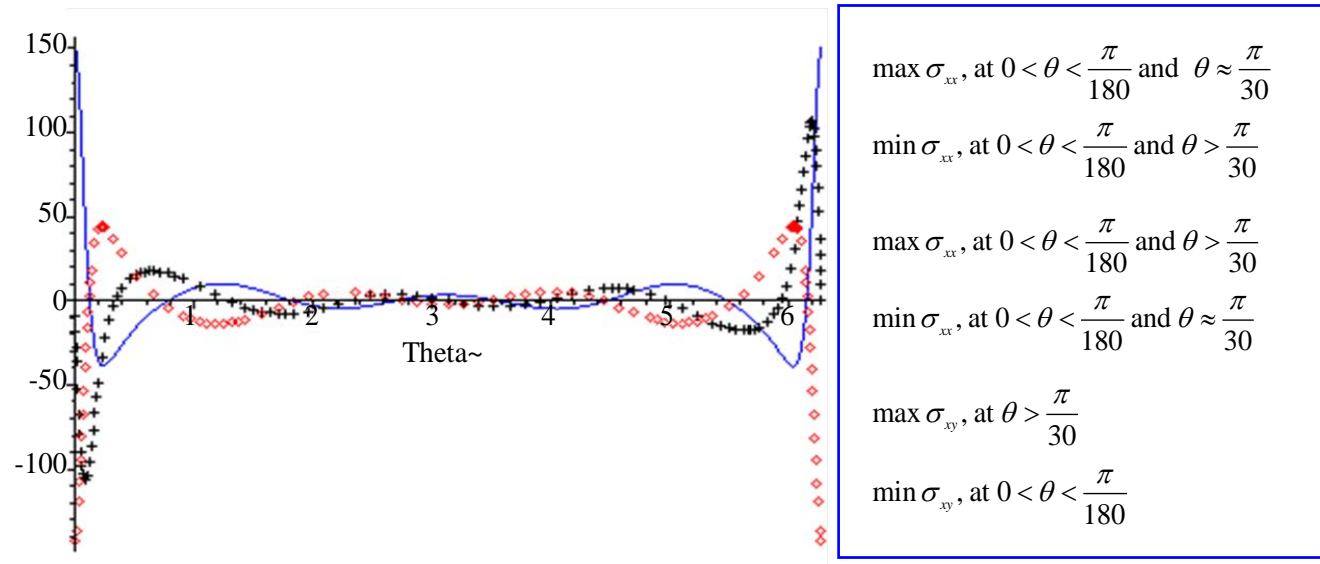

Figure 5. The relation between components of stresses and the angle made on the x-axis. $\sigma_{x x}$ ooooooooo;
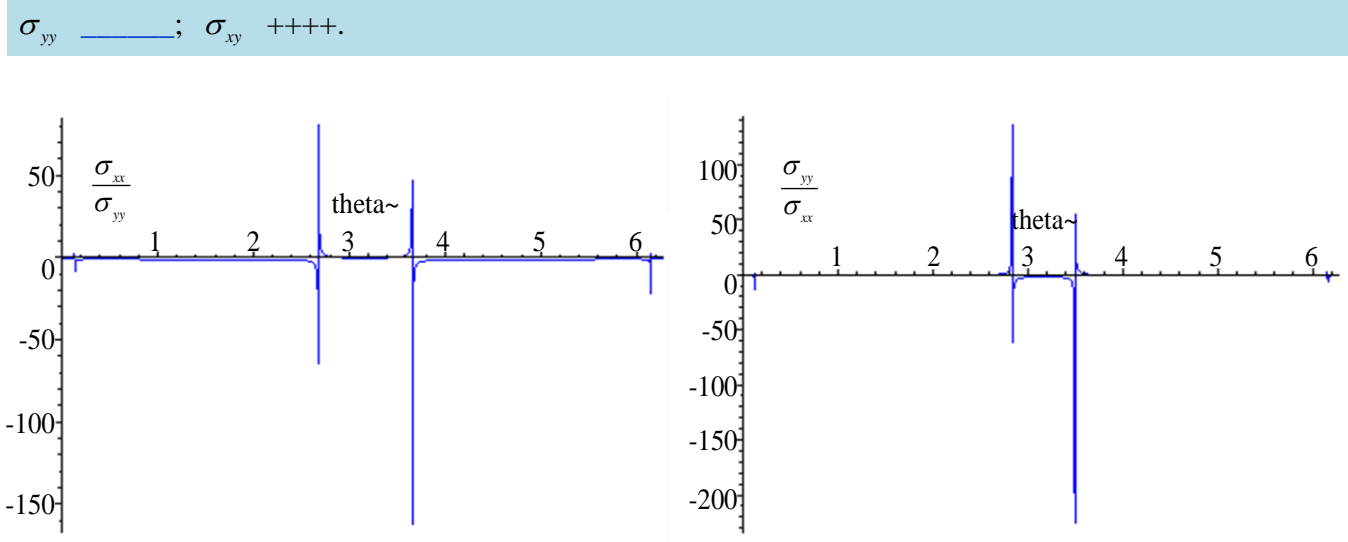

Figure 6. The ratio of vertical to horizontal stresses. 


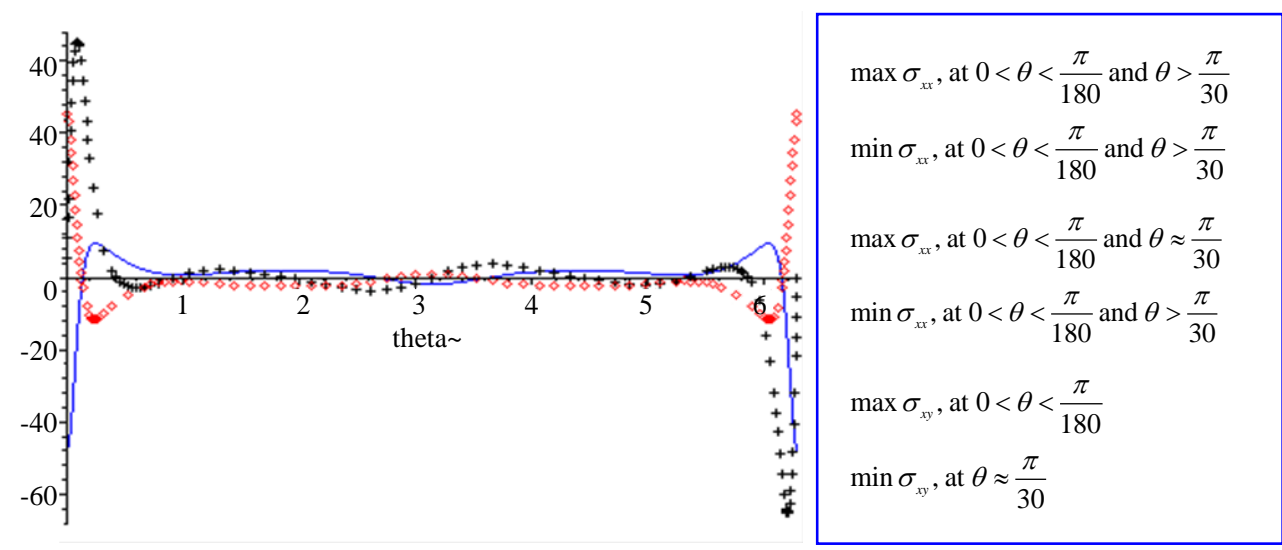

Figure 7. The relation between components of stresses and the angle made on the x-axis. $\sigma_{x x}$ 0000000000000; $\sigma_{y y} \longrightarrow ; \sigma_{x y}++++$.
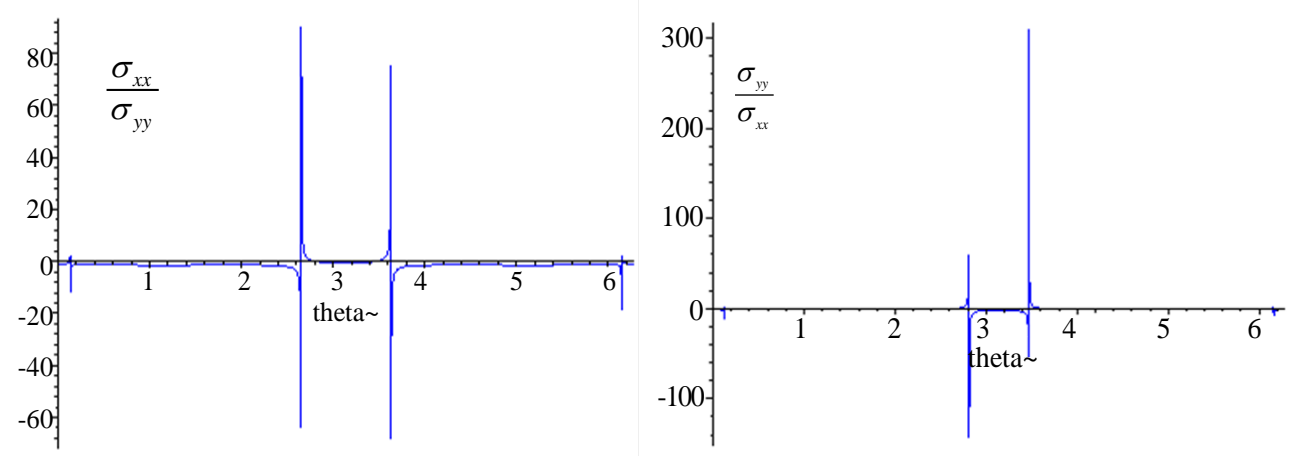

Figure 8. The ratio of vertical to horizontal stresses.

\section{Application 2: Bi-axial tension}

For $K=k, \Gamma=\bar{\Gamma}=\frac{p}{2}, \Gamma^{*}=0,0 \leq \theta \leq 2 \pi, X=Y=0$ and $f(t)=2 i \mu t$,

Goursat's functions in Equations (45) and (50) take the form

$$
\begin{gathered}
\phi(\zeta)=\frac{c P}{2} \sum_{j=1}^{2} \frac{h_{j}}{\left(k+h_{j} d_{j}\right)\left(\zeta-n_{j}\right)} \\
\psi(\zeta)=\frac{c P}{2} k \zeta^{-1}-\frac{\omega\left(\zeta^{-1}\right)}{\omega^{\prime}(\zeta)} \phi_{*}(\zeta)+\sum_{j=1}^{2} \frac{h_{j} \zeta}{1-n_{j} \zeta} \phi_{*}\left(n_{j}^{-1}\right)
\end{gathered}
$$

where,

$$
\phi_{*}(\zeta)=\phi^{\prime}(\zeta)+\frac{c P}{4} .
$$

This application discusses the first fundamental boundary value problem of an infinite layer stretched at infinity by the application of a uniform tensile stress of intensity heat in the negative direction of $y$-axis. This layer is weakened by a curvilinear hole $C$ which is free from stress.

For $n_{1}=0.09, n_{2}=0.4, m=-0.8, p=0.25$, the stress components $\sigma_{x x}, \sigma_{y y}$ and $\sigma_{x y}$ are obtained in large forms calculated by computer and illustrated in the following two cases:

1) In the thermoelasticity layer, we have the following shapes for the stress components by using the substitutions $G=0.5, q=0.1, \quad r_{0}=0.75, \alpha=0.7, v=0.7$, see Figure 9 and Figure 10. 


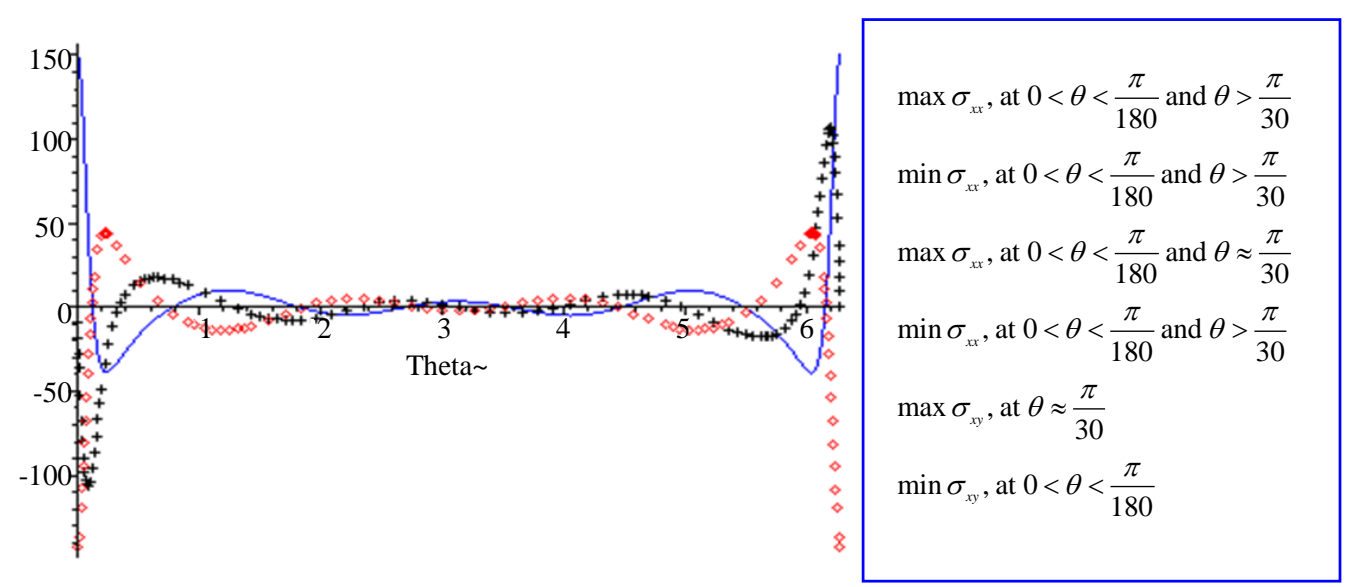

Figure 9. The relation between components of stresses and the angle made on the x-axis. $\sigma_{x x}$ 0000ooooo; $\sigma_{y y} \longrightarrow \sigma_{x y}++++$.
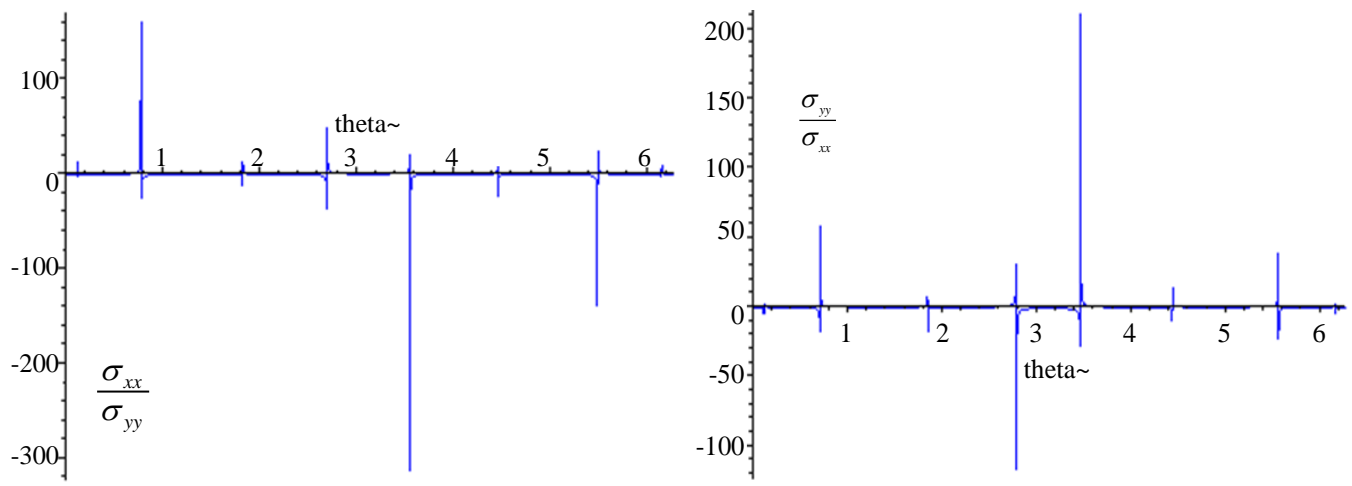

Figure 10. The ratio of vertical to horizontal stresses.

2) When the study is in the normal layer, we have the following shapes for the stress components, see Figure 11 and Figure 12.

3. Application 3: When a couple with a given moment acting on the curvilinear hole and the stresses vanishing at infinity

For $K=k, \Gamma=\frac{P}{4}, \Gamma^{*}=\frac{-P}{2} \mathrm{e}^{-2 i \theta}, 0 \leq \theta \leq 2 \pi, X=Y=0$ and $f=2 i \mu \varepsilon t$,

Goursat's functions in Equations (45), (50) take the form

$$
\begin{gathered}
\phi(\zeta)=2 c \mu \varepsilon i \sum_{j=1}^{2} \frac{\left(m+n_{j}^{2}\right)}{\left(k-h_{j} d_{j}\right)\left(\zeta-n_{j}\right)} \\
\psi(\zeta)=-\frac{\omega\left(\zeta^{-1}\right)}{\omega^{\prime}(\zeta)} \phi^{\prime}(\zeta)+\sum_{j=1}^{2}\left[2 i c \mu \varepsilon\left(n_{j}+\zeta^{-1}\right)+\frac{h_{j} \zeta}{1-n_{j} \zeta} \phi^{\prime}\left(n_{j}^{-1}\right)\right]
\end{gathered}
$$

where,

$$
\begin{aligned}
& \varepsilon=\frac{M}{4 \pi c \mu} \sum_{j=1}^{2}\left[\frac{n_{j}^{2}\left(m+n_{j}^{2}\right)-k\left(1-n_{j}^{2}(m+2)\right)}{m\left(m+n_{j}^{2}\right)+k\left(1-n_{j}^{2}(m+2)\right)}\right], \\
& M=c \pi p \sum_{j=1}^{2}\left(m+n_{j}^{2}\right)\left(\frac{k+1}{k-h_{j} d_{j}}\right) \sin 2 \theta .
\end{aligned}
$$




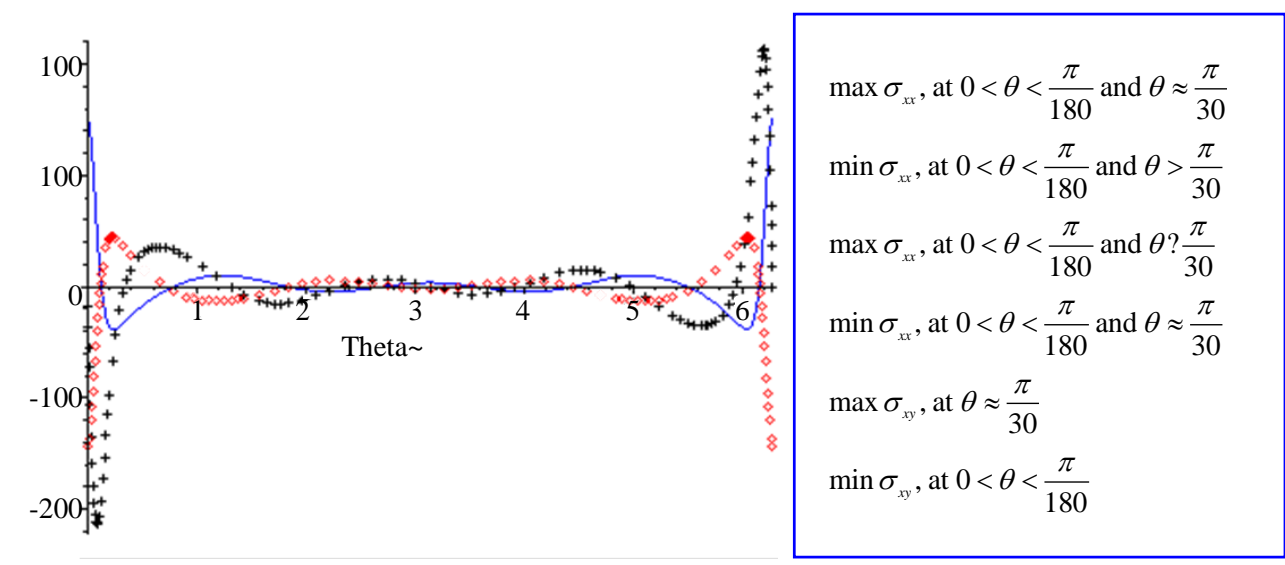

Figure 11. The relation between components of stresses and the angle made on the x-axis. $\sigma_{x x}$ 0000000000000; $\sigma_{y y} \longrightarrow ; \sigma_{x y}++++$.

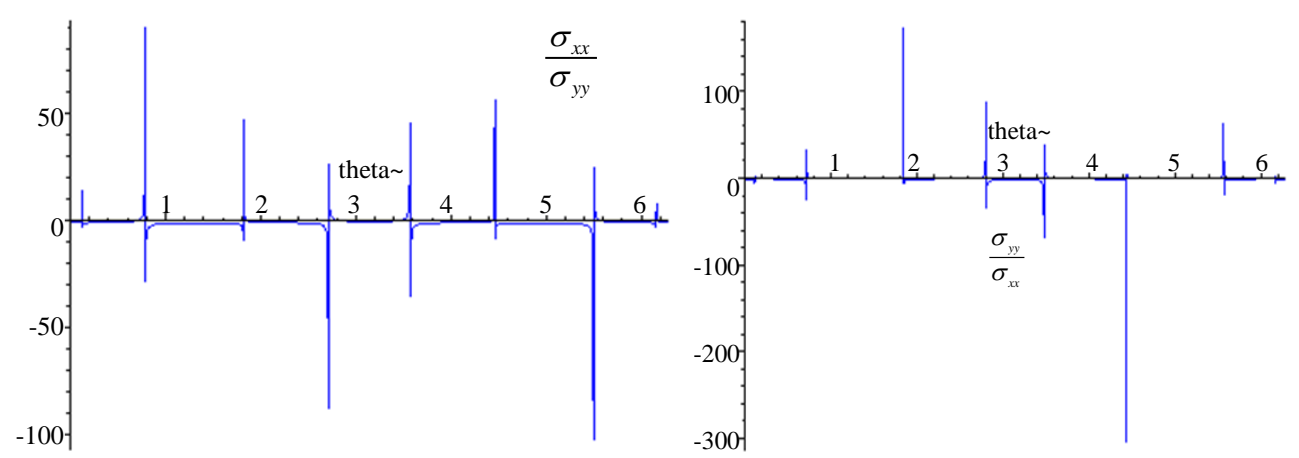

Figure 12. The ratio of vertical to horizontal stresses.

This application discusses the first fundamental boundary value problem of an infinite layer stretched at infinity by the application of a uniform tensile stress of intensity heat in the negative direction of $y$-axis. This layer is weakened by a curvilinear hole $C$ which is free from stress.

For $n_{1}=0.09, n_{2}=0.4, m=-0.8, p=0.25$, the stress components $\sigma_{x x}, \sigma_{y y}$ and $\sigma_{x y}$ are obtained in large forms calculated by computer and illustrated in the following two cases:

1) In the thermoelasticity layer, we have the following shapes for the stress components by using the substitutions $G=0.5, q=0.1, r_{0}=0.75, \alpha=0.7, v=0.7$, see Figure 13 and Figure 14 .

2) When the study is in the normal layer, we have the following shapes for the stress components, see Figure 15 and Figure 16.

\section{Application 4: The external force acts on the center of the curvilinear hole}

For $K=k, \Gamma=\Gamma^{*}=f=0$, Then, Goursat, s functions in Equations (45), (50) take the form

$$
\begin{gathered}
\phi(\zeta)=\frac{1}{2 \pi(1+k)} \sum_{j=1}^{2} \frac{h_{j} n_{j}^{v}}{\zeta^{\ell}-n_{j}}\left[\frac{k h_{j} d_{j}(X+i Y)}{k^{2}-h_{j}^{2} d_{j}^{2}}-\left\{\frac{h_{j}^{2} d_{j}^{2}}{k^{2}-h_{j}^{2} d_{j}^{2}}+1\right\}(X-i Y)\right] \\
\psi(\zeta)=-\frac{\omega\left(\zeta^{-1}\right)}{\omega^{\prime}(\zeta)} \phi_{*}(\zeta)+\sum_{j=1}^{2} \frac{h_{j} \zeta^{\ell}}{1-n_{j} \zeta^{\ell}} \phi_{*}\left(n_{j}^{-1}\right)
\end{gathered}
$$

where,

$$
\phi_{*}(\zeta)=\phi^{\prime}(\zeta)-\frac{X+i Y}{2 \pi(1+k) \zeta}
$$




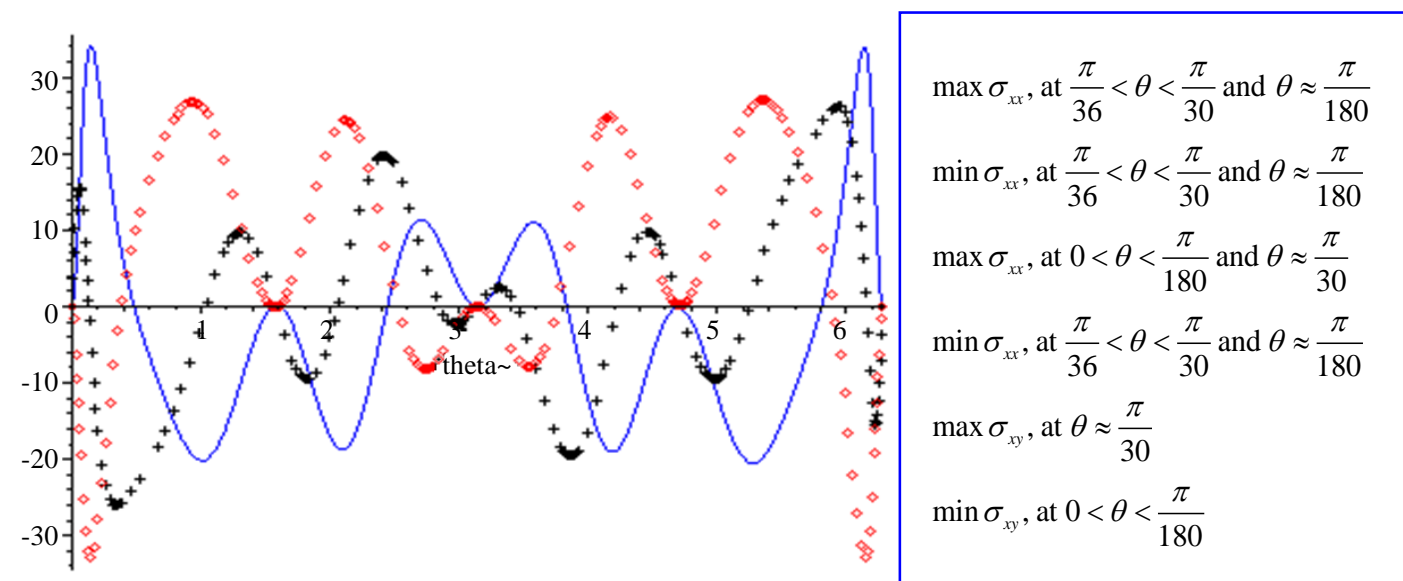

Figure 13. The relation between components of stresses and the angle made on the x-axis. $\sigma_{x x}$ oooooooooooo; $\sigma_{y y}$
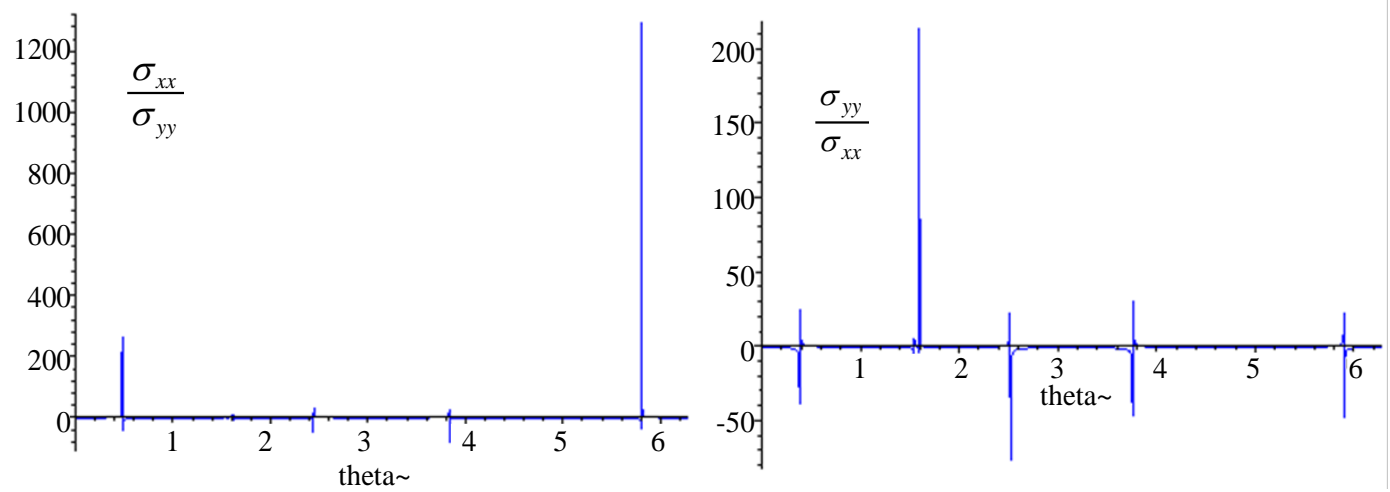

Figure 14. The ratio of vertical to horizontal stresses.

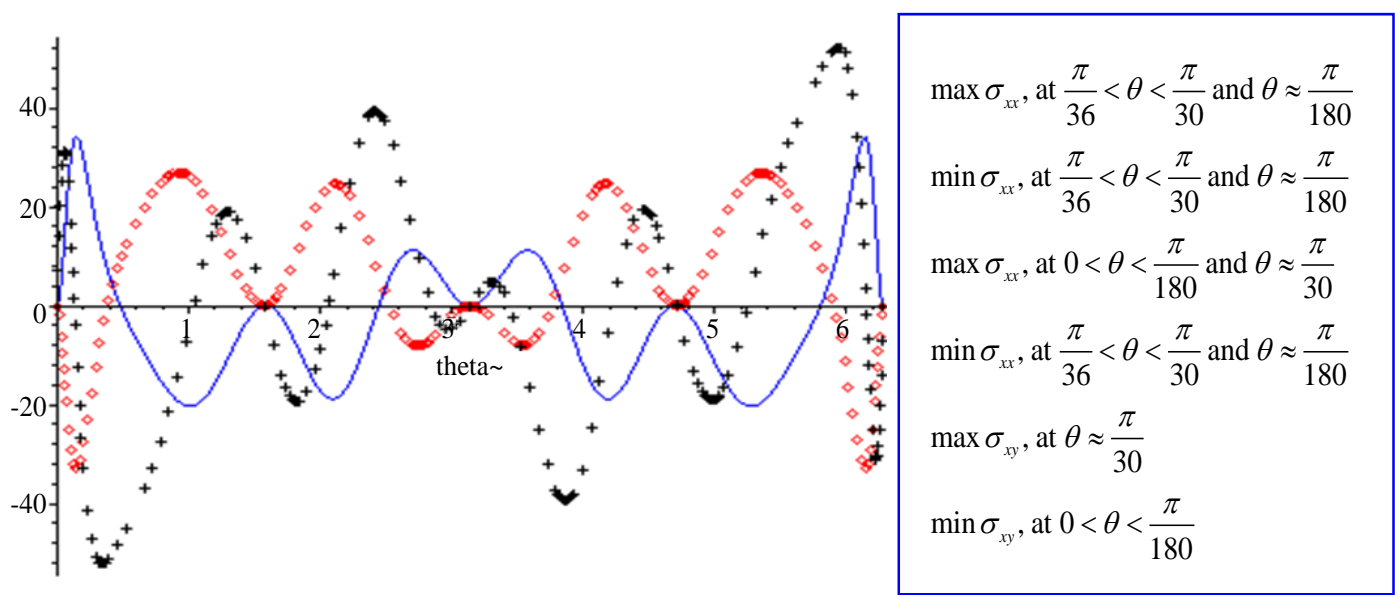

Figure 15. The relation between components of stresses and the angle made on the x-axis. $\sigma_{x x}$ oooooooooooo; $\sigma_{y y} \longrightarrow \sigma_{x y}++++$.

This application discusses the first fundamental boundary value problem of an infinite layer stretched at infinity by the application of a uniform tensile stress of intensity heat in the negative direction of y-axis. This layer is weakened by a curvilinear hole $C$ which is free from stress. 
For $n_{1}=-0.2, n_{2}=0.2, m=0.1, \ell=5, p=0.25$, the stress components $\sigma_{x x}, \sigma_{y y}$ and $\sigma_{x y}$ are obtained in large forms calculated by computer and illustrated in the following two cases:

1) In the thermoelasticity layer, we have the following shapes for the stress components by using the substitutions $G=0.5, q=0.1, \quad r_{0}=0.75, \alpha=0.7, \quad v=0.7$, see Figure 17 and Figure 18 .

2) When the study is in the normal layer, we have the following shapes for the stress components, see Figure 19 and Figure 20.

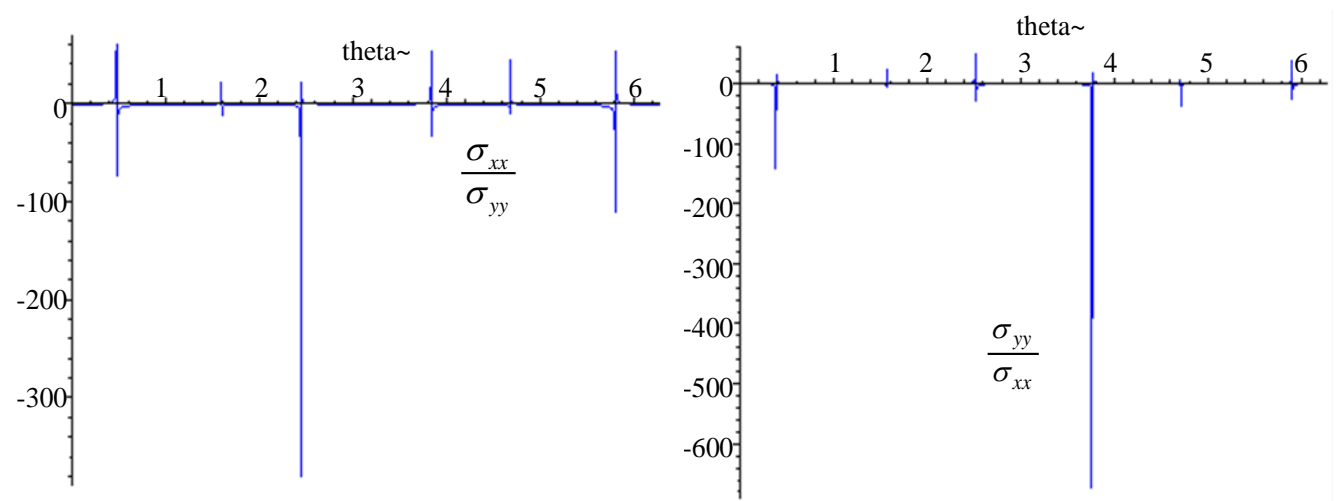

Figure 16. The ratio of vertical to horizontal stresses.

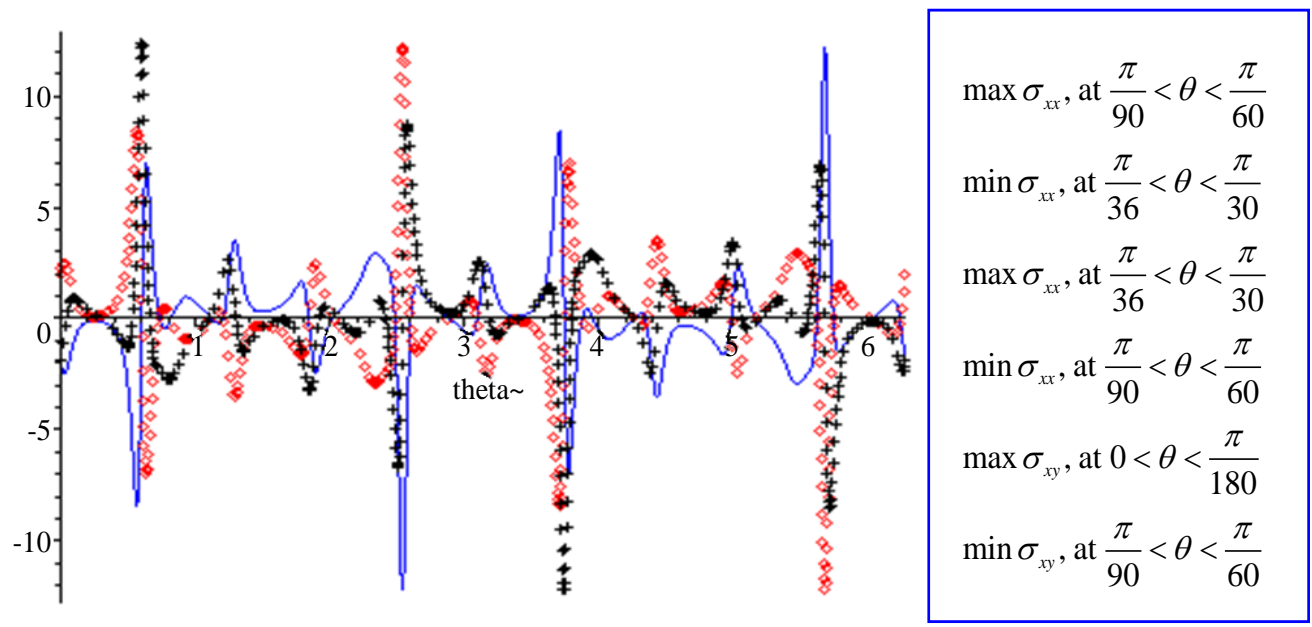

Figure 17. The relation between components of stresses and the angle made on the x-axis. $\sigma_{x x}$ 0000000000000; $\sigma_{y y} \longrightarrow \sigma_{x y}++++$.
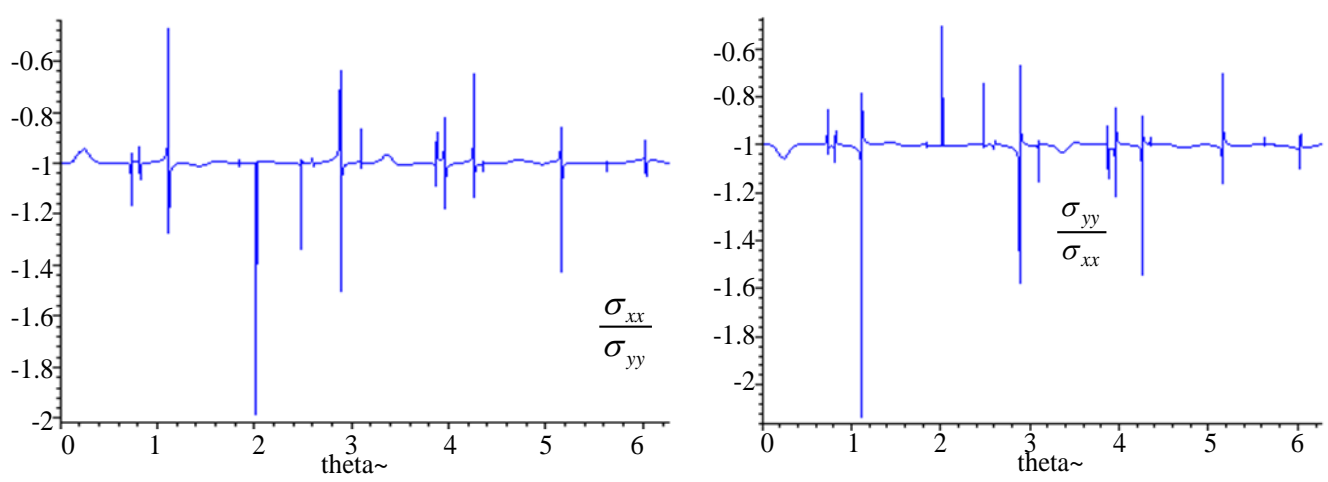

Figure 18. The ratio of vertical to horizontal stresses. 


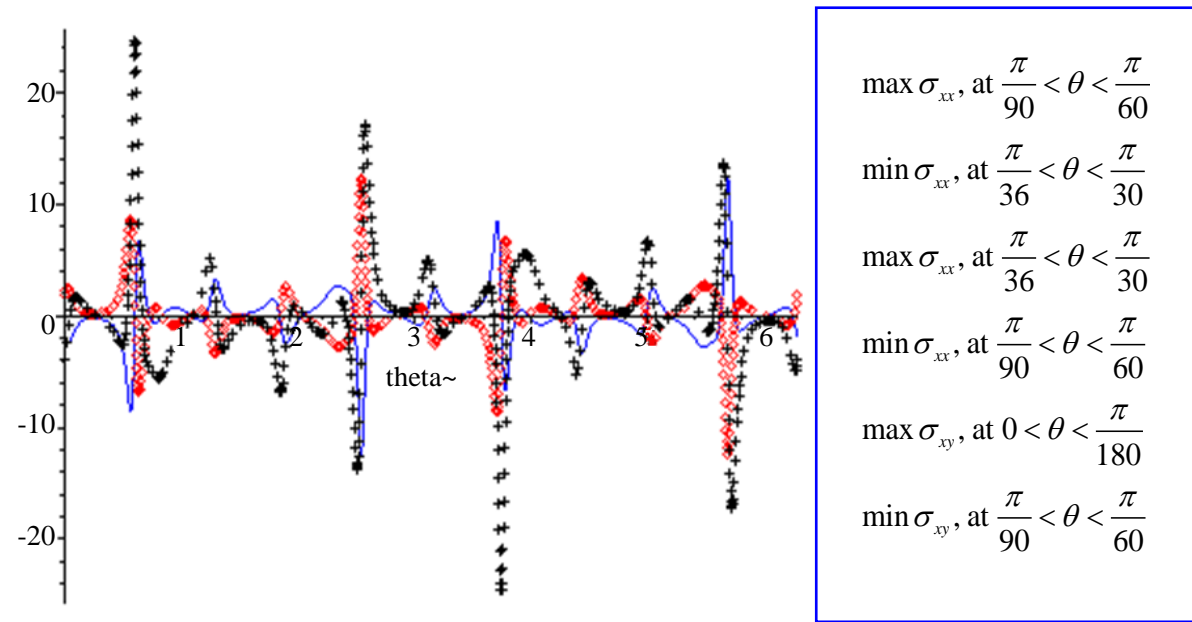

Figure 19. The relation between components of stresses and the angle made on the x-axis. $\sigma_{x x}$ 0000000000000; $\sigma_{y y} \longrightarrow ; \sigma_{x y}++++$.

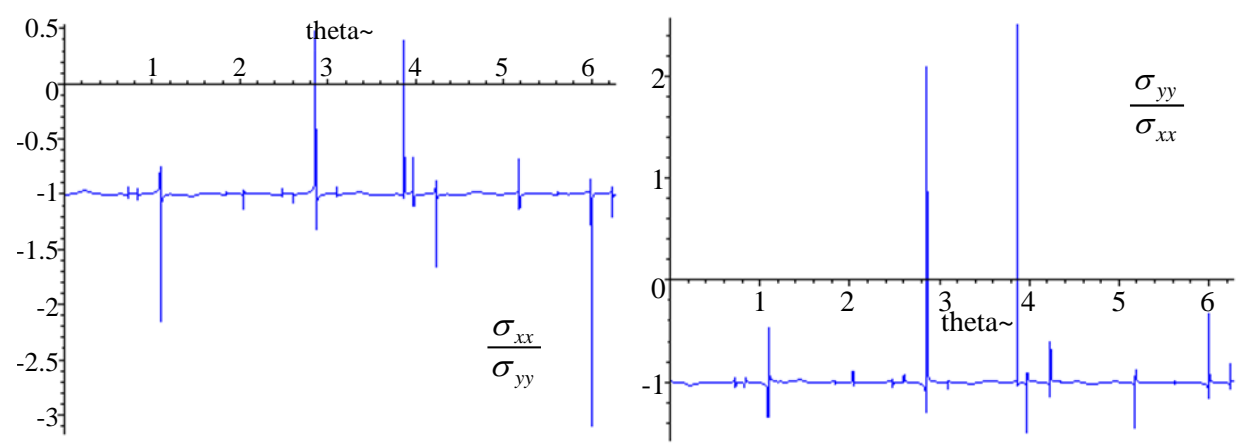

Figure 20. The ratio of vertical to horizontal stresses.

\section{Conclusions}

From the previous discussions we have the following results:

1) We find that the effect of heat is very clear; we find that the values of components stress are reduced with existence of heat, while at absence of heat we find the values of components of stresses are increasing.

2) With increasing angle and with absence $f(t)$, we find that

$$
\max \left(\frac{\sigma_{x x}}{\sigma_{y y}}\right)^{N} \text { and }\left(\frac{\sigma_{y y}}{\sigma_{x x}}\right)^{N}>\max \left(\frac{\sigma_{x x}}{\sigma_{y y}}\right)^{H} \text { and }\left(\frac{\sigma_{y y}}{\sigma_{x x}}\right)^{H}
$$

and,

$$
\min \left(\frac{\sigma_{x x}}{\sigma_{y y}}\right)^{N} \text { and }\left(\frac{\sigma_{y y}}{\sigma_{x x}}\right)^{N}<\min \left(\frac{\sigma_{x x}}{\sigma_{y y}}\right)^{H} \text { and }\left(\frac{\sigma_{y y}}{\sigma_{x x}}\right)^{H} \text {. }
$$

3) With increasing angle and with existence $f(t)$, we find that

$$
\max \left(\frac{\sigma_{x x}}{\sigma_{y y}}\right)^{N} \text { and }\left(\frac{\sigma_{y y}}{\sigma_{x x}}\right)^{N}<\max \left(\frac{\sigma_{x x}}{\sigma_{y y}}\right)^{H} \text { and }\left(\frac{\sigma_{y y}}{\sigma_{x x}}\right)^{H}
$$

and, 


$$
\min \left(\frac{\sigma_{x x}}{\sigma_{y y}}\right)^{N} \text { and }\left(\frac{\sigma_{y y}}{\sigma_{x x}}\right)^{N}>\min \left(\frac{\sigma_{x x}}{\sigma_{y y}}\right)^{H} \text { and }\left(\frac{\sigma_{y y}}{\sigma_{x x}}\right)^{H} .
$$

4) The components of stresses effected are clear by increasing the values of $\ell$.

\section{References}

[1] England, A.H. (1971) Complex Variable Methods in Elasticity. John Wiley Sons Ltd., London, New York.

[2] Kalandiya, A.I. (1975) Mathematical Method of Two-Dimensional Elasticity. Mir Publishers, Moscow.

[3] Sokolnikff, I.S. (1950) Mathematical Theory of Elasticity. New York.

[4] Abd-Alla, A.M. (2013) Journal of Computational and Theoretical Nanoscience, 10, 1914-1920. http://dx.doi.org/10.1166/jctn.2013.3148

[5] Narayanan, M. and Peter, A.J. (2012) Quantum Matter, 1, 53-58. http://dx.doi.org/10.1166/qm.2012.1005

[6] Bhattacharya, P.S. and Ghatak, K.P. (2012) Quantum Matter, 1, 63-85. http://dx.doi.org/10.1166/qm.2012.1007

[7] Kalandiya, A.I. (1975) Mathematical Theory of Elasticity. Mir Publishers, Moscow.

[8] Muskhelishvili, N.I. (1953) Some Basic Problems of Mathematical Theory of Elasticity. Noordhoff International, Leyden.

[9] Abdou, M.A. and Kkhar El-din, E.A. (1994) Journal of Computational and Applied Mathematics, 56, 341-361. http://dx.doi.org/10.1016/0377-0427(94)90088-4

[10] Abdou, M.A. (2002) Applied Mathematics and Computation, 125, 177-193. http://dx.doi.org/10.1016/S0096-3003(00)00118-1

[11] Abdou, M.A. and Asseri, S.A. (2009) Journal of Thermal Stresses, 32, 1126-1148.

[12] Abdou, M.A. and Asseri, S.A. (2009) Applied Mathematics and Computation, 212, 23-36. http://dx.doi.org/10.1016/j.amc.2009.01.079

[13] Sabbah, A.S., Abdou, M.A. and Ismail, A.S. (2002) Proceeding Mathematical Physics Society Egypt, 34, $15-27$.

[14] Parkus, H. (1976) Thermoelasticity. Springer Verlag, Berlin. http://dx.doi.org/10.1007/978-3-7091-8447-9

[15] El-Sirafy, I.H. and Abdou, M.A. (1984) Journal of Mathematical and Physical Sciences, 18, 205-214.

[16] Abdou, M.A. and Khamis, A.K. (2000) Bulletin of Calcutta Mathematical Society, 29, 313-326.

[17] El-Naggar, A.M., Kishka, Z., Abd-Alla, A.M., Abbas, I.A., Abo-Dahab, S.M. and Elsagheer, M. (2013) Journal of Computational and Theoretical Nanoscience, 10, 1408-1417. http://dx.doi.org/10.1166/jctn.2013.2862

[18] Abd-Alla, A.M. and Yahya, G.A. (2013) Journal of Computational and Theoretical Nanoscience, 10, 750-755. http://dx.doi.org/10.1166/jctn.2013.2765

[19] Abd-Alla, A.M., Abo-Dahab, S.M., Al-Thamali, T.A. and Mahmoud, S.R. (2013) Journal of Computational and Theoretical Nanoscience, 10, 297-305. http://dx.doi.org/10.1166/jctn.2013.2695

[20] Abd-Alla, A.M., Yahya, G.A. and El-Thagafy, M.H. (2013) Journal of Computational and Theoretical Nanoscience, 10, 347-352. http://dx.doi.org/10.1166/jctn.2013.2703

[21] Abd-Alla, A.M., Yahya, G.A. and Mahmoud, S.R. (2013) Journal of Computational and Theoretical Nanoscience, 10, 455-463. http://dx.doi.org/10.1166/jctn.2013.2718

[22] Abd-Alla, A.M. and Ahmed, S.M. (1996) Earth, Moon, and Planets, 75, 185-197. http://dx.doi.org/10.1007/BF02592996

[23] Abd-Alla, A.M., Abo-Dahab, S.M. and Al-Thamali, T.A. (2012) Journal of Mechanical Science and Technology, 26, 2815-2823. http://dx.doi.org/10.1007/s12206-012-0736-5

[24] Bose, P.K., Paitya, N., Bhattacharya, S., De, D., Saha, S., Chatterjee, K.M., Pahari, S. and Ghatak, K.P. (2012) Quantum Matter, 1, 89-126. http://dx.doi.org/10.1166/qm.2012.1009

[25] Tüzün, B. and Erkoç, C. (2012) Quantum Matter, 1, 136-148. http://dx.doi.org/10.1166/qm.2012.1012

[26] Ono, T., Fujimoto, Y. and Tsukamoto, S. (2012) Quantum Matter, 1, 4-19. http://dx.doi.org/10.1166/qm.2012.1002

[27] Muskhelishvili, N.I. (1949) Some Basic Problems of the Mathematical Theory of Elasticity. Nauka, Moscow.

[28] Abdou, M.A. (1994) Alexandria Engineering Journal, 33, 227.

[29] Abdou, M.A. and Badr, A.A. (1999) Journal of the Indian Academy of Wood Science, 51, 155-166. 